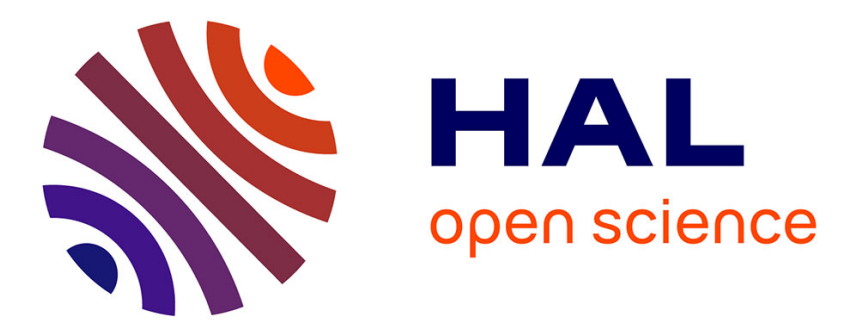

\title{
Magnetic flux distortion in two-phase liquid metal flow: Model experiment
}

Mithlesh Kumar, Wladimir Bergez, Philippe Tordjeman, Richard Arinero, Kevin Paumel

\section{- To cite this version:}

Mithlesh Kumar, Wladimir Bergez, Philippe Tordjeman, Richard Arinero, Kevin Paumel. Magnetic flux distortion in two-phase liquid metal flow: Model experiment. Journal of Applied Physics, 2016, vol. 119 ( $\left.\mathrm{n}^{\circ} 18\right)$, pp. 185105/1-185105/7. 10.1063/1.4950792 . hal-01561662

\section{HAL Id: hal-01561662 \\ https://hal.science/hal-01561662}

Submitted on 13 Jul 2017

HAL is a multi-disciplinary open access archive for the deposit and dissemination of scientific research documents, whether they are published or not. The documents may come from teaching and research institutions in France or abroad, or from public or private research centers.
L'archive ouverte pluridisciplinaire HAL, est destinée au dépôt et à la diffusion de documents scientifiques de niveau recherche, publiés ou non, émanant des établissements d'enseignement et de recherche français ou étrangers, des laboratoires publics ou privés. 


\section{Open Archive TOULOUSE Archive Ouverte (OATAO)}

OATAO is an open access repository that collects the work of Toulouse researchers and makes it freely available over the web where possible.

This is an author-deposited version published in : http://oatao.univ-toulouse.fr/ Eprints ID : 18058

To link to this article : DOI:10.1063/1.4950792

URL : $\underline{\text { http://dx.doi.org/10.1063/1.4950792 }}$

To cite this version : Kumar, Mithlesh and Bergez, Wladimir and Tordjeman, Philippe and Arinero, Richard and Paumel, Kevin Magnetic flux distortion in two-phase liquid metal flow: Model experiment. (2016) Journal of Applied Physics, vol. 119 ( $\left.\mathrm{n}^{\circ} 18\right)$. pp. 185105/1-185105/7. ISSN 0021-8979

Any correspondence concerning this service should be sent to the repository administrator: staff-oatao@,listes-diff.inp-toulouse.fr 


\title{
Magnetic flux distortion in two-phase liquid metal flow: Model experiment
}

\author{
M. Kumar, ${ }^{1,2}$ W. Bergez, ${ }^{1}$ Ph. Tordjeman, ${ }^{1, a)}$ R. Arinero, ${ }^{3}$ and K. Paumel ${ }^{2}$ \\ ${ }^{1}$ Université de Toulouse, Institut de Mécanique des Fluides de Toulouse, Allée du Professeur Camille Soula, \\ 31400 Toulouse, France \\ ${ }^{2}$ Nuclear Technology Department, CEA, DEN, F-13108 Saint Paul Lez Durance, France \\ ${ }^{3}$ Université Montpellier, IES, UMR 5214, F-34000 Montpellier, France
}

\begin{abstract}
In this paper, we present the model experiments in order to study the magnetic flux distortion of a two-phase liquid metal flow excited by an AC magnetic field in a range of pulsation where Faraday induction and Lorentz force effects are significant. These experiments realized with solid aluminum rods allow to characterize the effects of flow velocity $\left(0 \leq U \leq 1 \mathrm{~ms}^{-1}\right)$, void fraction $(0 \leq \alpha \leq 6.9 \%)$, pulsation of the AC magnetic field $\left(1.5 \times 10^{3} \leq \omega \leq 12.5 \times 10^{3} \mathrm{rad} \mathrm{s}^{-1}\right)$, and of two different void geometries. The results are analyzed on the basis of a first order expansion of magnetic flux in $U$ and $\alpha$. Despite the strong coupling between Faraday induction and Lorentz force effects, the results show that the contributions of $U$ and $\alpha$ on a magnetic flux distortion can be well separated at both low magnetic Reynolds number and $\alpha$ values. These results are independent of void geometry.

[http://dx.doi.org/10.1063/1.4950792]
\end{abstract}

\section{INTRODUCTION}

The fundamental question of how the magnetic flux is perturbed by a non-conducting dispersed phase in liquid metal flow is an open question. This problem arises in industrial processes as in nuclear engineering of Sodium Cooled Fast Reactors or in metallurgy. In the presence of a variable magnetic flux density $\vec{B}$, the Lorentz force in momentum conservation equation depends on the electrical current density $\vec{J}$ that results from the Faraday and flow induced Eddy currents: $\vec{J}=\sigma(\vec{E}+\vec{U} \times \vec{B})$, where the electric field $\vec{E}$ comes from the Maxwell-Faraday equation $\nabla \times \vec{E}=-\partial \vec{B} / \partial t$, and $\vec{U}$ is the fluid velocity. The Eddy currents generate a perturbation in the magnetic fields that modify the total magnetic flux, $\phi=\int \vec{B} \cdot \mathrm{d} \vec{s}$. In the presence of two-phase flow, $\phi$ is affected by the void volume fraction $\alpha$ of the non-conducting phase with the coupling effects involving the electrical conductivity, the flow velocity, and the electromagnetic field distribution. Indeed, due to the strong coupling between the Maxwell's equations, hydrodynamic equations and the Ohm's law, the presence of void modifies all the field quantities both at the local scale and at the $\phi$ integrated scale.

In the literature, we find different approaches to model the $\alpha$ and velocity effects on the magnetic flux. Historically, Maxwell established by homogenization, the equivalent electrical conductivity of a static two-phase medium at small $\alpha .{ }^{1}$ This model is based on the multipolar expansion of the electrical potential of small spheres diluted in a continuous medium of electrical conductivity $\sigma_{0}$ and gives at first order $\sigma(\alpha) \approx \sigma_{0}\left(1-\frac{3}{2} \alpha\right)$. This model predicts a linear dependence in $\alpha$, which has been confirmed experimentally. ${ }^{2}$ However, experiments point out that the magnetic

\footnotetext{
${ }^{\text {a)} E l e c t r o n i c ~ m a i l: ~ p h i l i p p e . t o r d j e m a n @ i m f t . f r ~}$
}

flux is strongly perturbed by a diluted bubbly flow and cannot be modeled by the Maxwell approach., ${ }^{3,4}$ This result has been confirmed by numerical simulations. ${ }^{5}$ Using the reciprocity theorem and an expansion of magnetic vector potential, Bowler et al. have estimated the perturbation in impedance due to a single small defect in a conducting medium. ${ }^{6,7}$ This result is only valid for small skin depths and does not allow to predict the perturbation in magnetic flux due to the distribution of voids in the core of a conducting flow. For single phase conducting flow in a circular cylinder at constant or very slowly varying magnetic fields (i.e., $\omega \leq 100 \mathrm{rad} \mathrm{s}^{-1}$ ), the electrical potential is given by a Poisson equation: $\nabla^{2} V=\nabla \cdot(\vec{U} \times \vec{B}){ }^{8,9}$ In the presence of voids, some authors have extended this approach and showed for axisymmetric two-phase flow (homogeneous bubbly or annular flow) that the electrical potential varies as $V \sim 1 /(1-\alpha) \cdot{ }^{10,11}$ In the literature, $\alpha$ is generally defined as the relative flow rate of gas and not as the real volumic fraction, which is difficult to measure experimentally in liquid metal. ${ }^{3,10,12}$

The effects of Lorentz and buoyancy forces on the deformation and the velocity of a single gas bubble rising in a liquid metal column are well described in the literature. Indeed, for static and rotating magnetic fields, experiments and numerical simulations point out that the hydrodynamic forces are modified by the Eddy current distribution around the bubble, which consequently affects the trajectory, the velocity, and the shape of the bubble. ${ }^{13-20}$ On the other hand, for AC magnetic fields at moderate frequencies for which induction is not negligible in the core of the flow, the effects of void on Eddy current distribution are not well explained. ${ }^{3}$

In this paper, we present model experiments that show coupling effects between the Lorentz force and the Faraday induction on the Eddy current distribution in a model twophase liquid metal flow. In these experiments, we study the effects of velocity $U$, pulsation $\omega$, and the void fraction $\alpha$ at 
room temperature. The magnetic field $\vec{B}$ is generated by a primary coil through which the liquid metal flows at constant velocity. The magnetic flux is then measured with two coaxial secondary coils and integrates the distortion of the induced Eddy currents due to the presence of void in the conducting medium. All the results will be analyzed on the basis of a perturbation expansion model of the magnetic flux. ${ }^{21}$ The experimental results show that, at small magnetic Reynolds number $\operatorname{Re}_{\mathrm{m}}\left(\operatorname{Re}_{\mathrm{m}}=\sigma \mu_{0} U \delta\right.$, where $\delta$ is the penetration depth of the magnetic flux in the medium) and at small $\alpha, \phi$ is well approximated by a linear expansion. Hence, the $U$ and $\alpha$ contributions on $\phi$ are separated in two terms which in addition are found to be independent at first order.

The paper is organized as follows. In Section II, the perturbation magnetic flux model is recalled. The experiments are detailed in Section III and the results are discussed in Section IV. Conclusions are given in Section V.

\section{THEORY}

In this section, we recall the model that gives the perturbation of the magnetic flux, which can be measured with an Eddy current flowmeter (ECFM). ${ }^{21}$ This apparatus is well adapted to experiment the coupling between the Lorentz force effects and Faraday induction in a two-phase flow. Fig. 1 presents a sketch of the physical system which is modeled below.

A two-phase liquid metal with a void volume fraction $\alpha$ and characteristic velocity $U$ flows through a primary coil $P$. This coil is excited by an AC $I=I_{0} \cos \omega t$. Two coaxial secondary coils $\left(S_{1}\right.$ and $S_{2}$ ) measure the perturbation of the induced magnetic flux due to the Faraday and Lorentz force effects. The emf difference between the two secondary coils $\Delta V$ measures the total perturbed flux. In this problem, the magnetic Reynolds number $\mathrm{Re}_{\mathrm{m}}$ is based on the penetration depth of the magnetic field in the medium, $\delta=\sqrt{\frac{2}{\sigma \mu_{0} \omega}}$. Assuming a low $\operatorname{Re}_{\mathrm{m}}$ and coil diameter $D$ larger than $\delta, \vec{B}$ is weakly perturbed and the amplitude of the net magnetic flux crossing coils $S_{1}$ and $S_{2}$ is given at first order,

$$
\phi=\int \vec{B} \cdot \overrightarrow{d s} \approx \phi_{0}+\operatorname{Re}_{\mathrm{m}} \phi_{u}+\phi_{\alpha}(t) .
$$

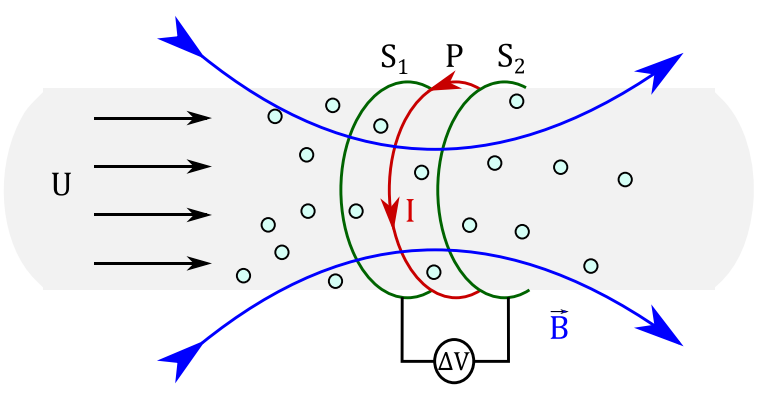

FIG. 1. Magnetic flux distortion system with a two-phase medium. $\mathrm{P}$ is the primary coil excited by an AC current $I, \mathrm{~S}_{1}$ and $\mathrm{S}_{2}$ are the two secondary coils, and $U$ is the velocity of the two-phase flow.
All the fluxes are spatially averaged over the length of the secondary coils. Ideally, $\phi=0$ for the well balanced $S_{1}$ and $S_{2}$ without motion and without void. $\phi_{0}$ is defined as the time average when $U$ vanishes: $\phi_{0}=\lim _{U \rightarrow 0}\left[\frac{U}{L} \int_{0}^{L / U} \phi d t\right]$, where $L$ is the length large enough for the integral to be independent of $L$. In this condition, the fluid properties become continuous. $\phi_{u}$ is the average flux due to the Lorentz force effects, and $\phi_{\alpha}$ is the perturbation of the total flux due to the dispersed phase and is related to the distortion of the induced Eddy currents. In case of periodic distribution of the dispersed phase, this term can be expanded in Fourier series,

$$
\phi_{\alpha}(t)=\psi_{\alpha} \cos \left(\omega_{\alpha} t+\theta\right)+\cdots,
$$

where the amplitude $\psi_{\alpha}$ depends on $\alpha$, and $\omega_{\alpha} \ll \omega$ characterizes the spatial distribution $\left(\omega_{\alpha}=U k_{\alpha}\right.$, where $k_{\alpha}$ is the wave number characteristic of the dispersed phase). The constitutive equation of the liquid is $\vec{J}=\sigma(\vec{E}+\vec{U} \times \vec{B})$. Based on the coupling between Maxwell-Faraday and the MaxwellAmpere equations, the magnetic field in the medium has two contributions, one in phase and one in quadrature with $I$. Hence, the total flux can be decomposed as $\phi(t)=\phi_{\|} \cos \omega t$ $+\phi_{\perp} \sin \omega t$, where

$$
\begin{aligned}
& \phi_{\|} \approx \phi_{0, \|}+\operatorname{Re}_{\mathrm{m}} \phi_{u, \|}+\psi_{\alpha, \|} \cos \left(\omega_{\alpha} t+\theta\right), \\
& \phi_{\perp} \approx \phi_{0, \perp}+\operatorname{Re}_{\mathrm{m}} \phi_{u, \perp}+\psi_{\alpha, \perp} \cos \left(\omega_{\alpha} t+\theta\right) .
\end{aligned}
$$

$\Delta V(t)$ is obtained by time derivation of $\phi(t):$ the square of the modulus is $\|\Delta V\|^{2}=\Delta V_{\|}^{2}+\Delta V_{\perp}^{2}$. Considering the first terms in $O\left(\operatorname{Re}_{\mathrm{m}}\right)\left(\operatorname{Re}_{\mathrm{m}} \ll 1\right)$,

$$
\begin{aligned}
\|\Delta V\|^{2} \approx & \omega^{2}\left\{\left\langle\phi_{0} \mid \phi_{0}\right\rangle+\frac{\left\langle\psi_{\alpha} \mid \psi_{\alpha}\right\rangle}{2}+2\left\langle\phi_{0} \mid \psi_{\alpha}\right\rangle \cos \left(\omega_{\alpha} t+\theta\right)\right. \\
& +\frac{\left\langle\psi_{\alpha} \mid \psi_{\alpha}\right\rangle}{2} \cos \left(2 \omega_{\alpha} t+2 \theta\right) \\
& \left.+2 \operatorname{Re}_{\mathrm{m}}\left[\left\langle\phi_{0} \mid \phi_{u}\right\rangle+\left\langle\phi_{u} \mid \psi_{\alpha}\right\rangle \cos \left(\omega_{\alpha} t+\theta\right)\right]\right\} .
\end{aligned}
$$

In this formula, $\phi_{i}$ is defined as a vector, $\phi_{i}=\left[\phi_{i, \|}, \phi_{i, \perp}\right]$. $\left\langle\phi_{i} \mid \phi_{j}\right\rangle$ represents the scalar product between the two vectors $i$ and $j$. In Equation (5), $\|\Delta V\|^{2}$ is expressed as a function of the dominant term in Fourier expansion. The last term in $\mathrm{Re}_{\mathrm{m}}$ corresponds to the magnetic flux component perturbed by the motion. We note that $\left\langle\phi_{0} \mid \phi_{0}\right\rangle$ and $\left\langle\psi_{\alpha} \mid \psi_{\alpha}\right\rangle$ appear at zero order. The terms $\left\langle\phi_{0} \mid \psi_{\alpha}\right\rangle,\left\langle\phi_{0} \mid \phi_{u}\right\rangle$, and $\left\langle\phi_{u} \mid \psi_{\alpha}\right\rangle$ represent the coupling effects of Faraday induction, Lorentz force, and dispersed phase. The term $\left\langle\phi_{u} \mid \phi_{u}\right\rangle$, which appears at second order in $\mathrm{Re}_{\mathrm{m}}$ has been neglected.

From the experiments and based on this model, the contributions of the dispersed phase and of the velocity on the net magnetic flux can be measured by the Fourier analysis of the $\|\Delta V\|^{2}$ signal. The amplitude of $\omega_{\alpha}$ term at zero velocity $\left(\left\langle\phi_{0} \mid \psi_{\alpha}\right\rangle\right)$ gives the perturbation due to Faraday induction effects. The second $\omega_{\alpha}$ term, which depends on $\operatorname{Re}_{\mathrm{m}}\left(\left\langle\phi_{u} \mid \psi_{\alpha}\right\rangle\right)$, exhibits the coupling effects between the velocity and the void fraction on the induced Eddy currents. Finally, the amplitude of $2 \omega_{\alpha}$ term $\left(\left\langle\psi_{\alpha} \mid \psi_{\alpha}\right\rangle\right)$ characterizes the influence of the dispersed phase and is independent of the Lorentz force effects. 


\section{EXPERIMENTAL SET UP}

The experimental set up is shown in Fig. 2. The primary coil $P$ of outer radius $20 \mathrm{~mm}$ and length $30 \mathrm{~mm}$ consists of 70 turns of copper wires wrapped around a non-conducting non-ferromagnetic structure. A frequency generator excites the $P$ coil by an AC $I=I_{0} \cos \omega t$. The pulsation $\omega$ varies between $1.5 \times 10^{3} \mathrm{rad} \mathrm{s}^{-1}$ and $12.5 \times 10^{3} \mathrm{rad} \mathrm{s}^{-1}$. This distortion in magnetic flux is monitored using the two coaxial secondary coils $S_{1}$ and $S_{2}$ of outer radii $20 \mathrm{~mm}$ and length $20 \mathrm{~mm}$ each. They consist of 50 turns of copper wires. The three coils constitute a standard ECFM sensor. The difference voltage $\Delta V=V_{2}-V_{1}$ between $S_{1}$ and $S_{2}$ can be measured with an oscilloscope. In order to understand the physical origin and the nature of the Eddy current distortion due to $U$ and $\alpha$, we used a Lockin Amplifier (HF2LI-MF, Zurich Instruments), which samples the in-phase and quadrature components of distorted flux with respect to the imposed current of the $P$ coil. The Lockin signal has a very high signal to noise ratio that allows to measure the $n V$ range signals.

A uniaxial displacement controller translates the coils at a given constant velocity $U$ in the range of $10^{-3}-1 \mathrm{~ms}^{-1}$. The conducting fluid is modeled by an aluminium rod along which the coils are moved. These experiments are analogous to a plug flow of a conducting liquid. In order to control the void fraction $\alpha$, grooves and holes are machined on the surface of the rod with spatial periodicity $\lambda_{\alpha}=2 \pi / k_{\alpha}$. Nine types of aluminium rods were used in the experiments to vary $\alpha$ between $0 \%$ and $6.9 \%$ and also to understand the effects of void geometry. All the dimensions are controlled after machining with a vernier caliper of precision $0.01 \mathrm{~mm}$.

The reference plain aluminium $\operatorname{rod}(\alpha=0 \%)$ has an outer radius of $19.25 \mathrm{~mm}$.

For groove-type rods which represent the geometrical void fractions $\alpha=0.3 \%, 2 \%, 4.5 \%$, and $6.9 \%$, respectively, $\lambda_{\alpha}=18.00 \mathrm{~mm}, 18.00 \mathrm{~mm}, 18.00 \mathrm{~mm}$, and $16.85 \mathrm{~mm}$, respectively; the depths of the grooves are $0.38 \mathrm{~mm}, 1.80 \mathrm{~mm}$, $3.20 \mathrm{~mm}$, and $4.23 \mathrm{~mm}$, respectively; the lengths of the grooves are $1.40 \mathrm{~mm}, 2.00 \mathrm{~mm}, 2.60 \mathrm{~mm}$, and $3.00 \mathrm{~mm}$, respectively; the outer radii of aluminium cylinder are $19.25 \mathrm{~mm}, 19.00 \mathrm{~mm}$, $19.00 \mathrm{~mm}$, and $19.25 \mathrm{~mm}$, respectively.

For hole-type (cylindrical bore-hole on outer surface) rods which represent the geometrical void fraction $\alpha=0.06 \%$, $0.22 \%, 0.54 \%$, and $1.62 \%$, respectively, the spatial frequencies of holes $\lambda_{\alpha}=17.45 \mathrm{~mm}$ each; the depths of the holes are $2.00 \mathrm{~mm}$ each; the diameter of the holes are $2.06 \mathrm{~mm}$, $3.88 \mathrm{~mm}, 6.03 \mathrm{~mm}$, and $10.21 \mathrm{~mm}$, respectively; the outer radii of aluminium cylinder are $19.25 \mathrm{~mm}$ each. The number of holes are fixed to achieve the given $\alpha$ values.

The experiments were realized at room temperature for which the electrical conductivity of the aluminium rod is $\sigma=3.8 \times 10^{7} \mathrm{~S} / \mathrm{m}$. The skin depth is then comprised between $5.2 \mathrm{~mm}$ and $1.8 \mathrm{~mm}$ for the experimental pulsation range. The amplitude of current intensity for all the experiments is in the range of $200-400 \mathrm{~mA}$. All the experimental results are given for a normalized intensity amplitude of $1 \mathrm{~A}$. Moreover, we have checked that the current remains constant within $0.1 \%$ during an experiment.

For each measurement, the ECFM was translated forward at a constant velocity, stopped, and translated back at the same velocity. This is repeated several times. Experiments with this system are very reproducible because the system is highly deterministic. Finally, we have verified that the electromagnetic background noise is not significant at these frequencies.

Fig. 3 displays a typical demodulated $\Delta V_{\|}$signal. This kind of signal can be obtained for in phase and quadrature components and also for $\|\Delta V\|$. Experimentally, $\phi_{0}$ is measured as the average value of the signal when there is very slow motion, typically $U=10^{-3} \mathrm{~m} / \mathrm{s}$ (inset of Fig. 3). In this case, the Lorentz force can be neglected. In motion at constant $U$, the average value of the signal is shifted with a value proportional to $\mathrm{Re}_{\mathrm{m}}$. In the presence of voids $(\alpha \neq 0 \%)$, this signal oscillates around this value. The amplitude of this oscillation gives $\psi_{\alpha}$. An example of the FFT spectrum of the fluctuations of $\|\Delta V\|^{2}$ signal is displayed in Fig. 4. For our experiments, all the FFT signals exhibit the two first prominent harmonic peaks in $\omega_{\alpha}$. This result validates the expansion at first order in $\mathrm{Re}_{\mathrm{m}}$ of the magnetic flux and also the Fourier

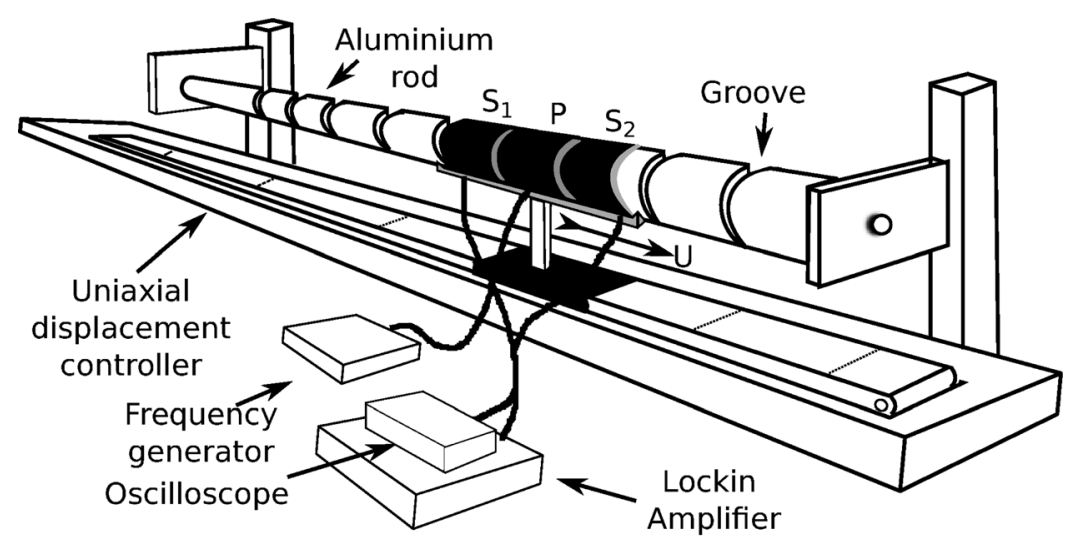

FIG. 2. Experimental set-up.

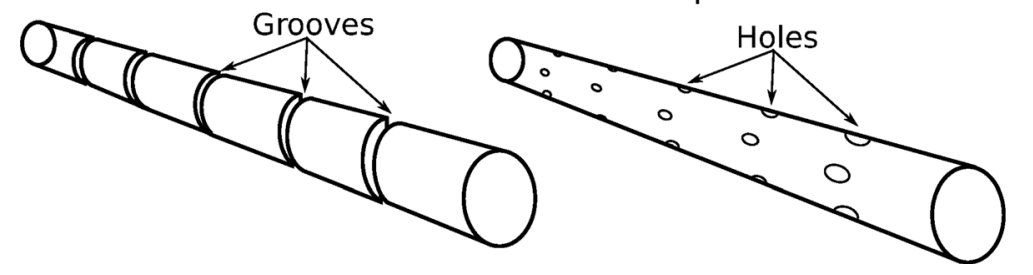




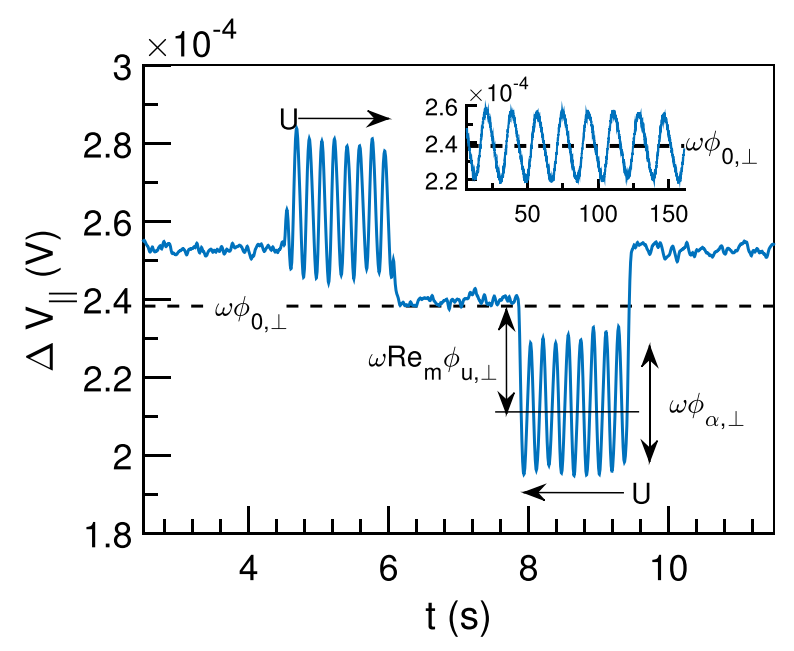

FIG. 3. Typical demodulated voltage difference of the two secondary coils, $\Delta V_{\|}$measured vs time for $\alpha=0.3 \%$ at $\omega=4712 \mathrm{rad} / \mathrm{s}$ and $U=0.1 \mathrm{~m} / \mathrm{s}$ (inset: $U=0.001 \mathrm{~m} / \mathrm{s}$ ).

series of the void fraction perturbation on the Eddy currents, Equations (1)-(2). The amplitude of the peak at $3 \omega_{\alpha}$ gives the first residue of Equation (5). The amplitude of the first peak is $\omega^{2} A_{1}=\omega^{2}\left[\left\langle\phi_{0} \mid \psi_{\alpha}\right\rangle+2 \operatorname{Re}_{\mathrm{m}}\left\langle\phi_{u} \mid \psi_{\alpha}\right\rangle\right]$ and the amplitude of the second peak is $\omega^{2} A_{2}=\omega^{2} \frac{\left\langle\psi_{\alpha} \mid \psi_{\alpha}\right\rangle}{2}$. We have verified that the value of $\sqrt{2 A_{2}}$ is equal to the norm of $\psi_{\alpha}$, which has been obtained from the direct FFT of $\phi_{\alpha}$ signal.

In summary, the experimental results were treated by two approaches: first, the in-phase and quadrature phase components of $\phi_{0}, \phi_{u}$, and $\phi_{\alpha}$ are directly measured from $\Delta V(t)$; second, $A_{1}, A_{2}$, and $\omega_{\alpha}$ were measured from the FFT of $\|\Delta V\|^{2}$. We have checked that the second approach is still valid for the non-periodic void distribution as long as $\omega_{\alpha}$ can be measured. By machining a specific rod with aperiodic grooves, we have observed that the intensity and the width of the two first harmonic peaks decreases and increases, respectively, when the spatial distribution of the grooves widens.

\section{RESULTS AND DISCUSSION}

Fig. 5 compares $\phi_{0, \|}(\omega)$ and $\phi_{0 \perp}(\omega)$ for $\alpha=0 \%, \alpha=$ $0.3 \%$ and $\alpha=6.9 \%$. We observe that $\phi_{0, \|}$ and $\phi_{0, \perp}$ are

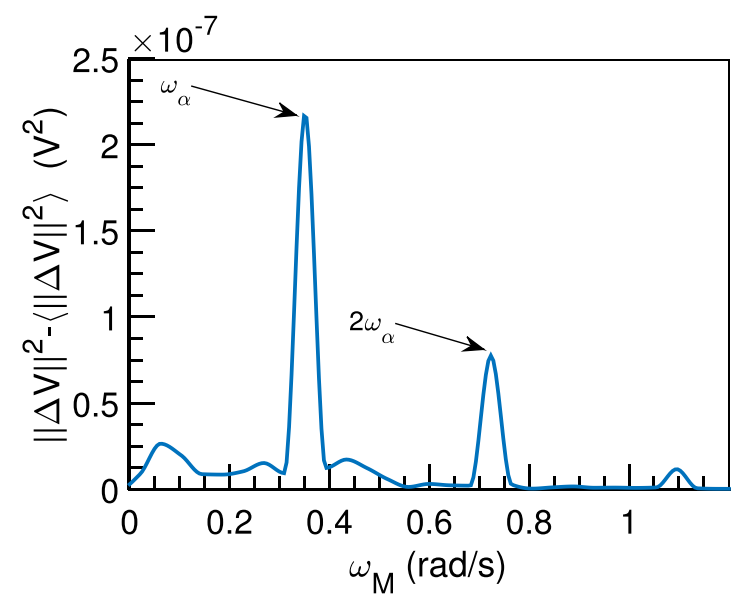

FIG. 4. FFT spectral density of fluctuations of $\|\Delta V\|^{2}$ vs the modulating pulsation $\omega_{\mathrm{M}}$ for $\alpha=6.9 \%$ and $U=0.001 \mathrm{~m} / \mathrm{s}$ at $6283 \mathrm{rad} / \mathrm{s}$ (data from [21]).

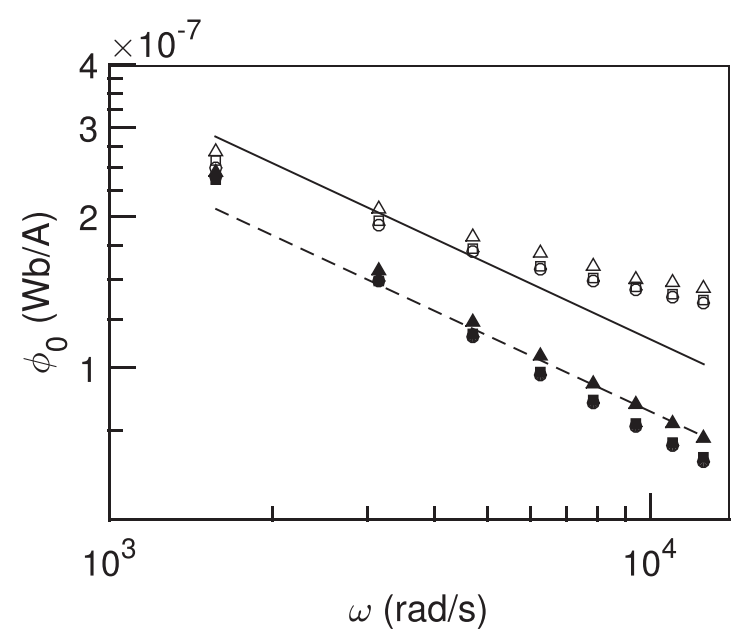

FIG. 5. $\phi_{0}$ vs $\omega$ at $U=0.001 \mathrm{~ms}^{-1}$ for $\alpha=0 \%(\bigcirc, \bigcirc), 0.3 \%(\square, \boldsymbol{\square}), 6.9 \%$ $(\triangle, \boldsymbol{\Delta})$; where $\phi_{0, \|}:$ unfilled symbols and $\phi_{0, \perp}$ : filled symbols. Lines represent the power law in $\omega^{-1 / 2}$.

weakly dependent on $\alpha$. Furthermore, the results show that $\phi_{0, \|}>\phi_{0, \perp}$, whatever $\omega$. The frequency dependence of $\phi_{0, \perp}$ obeys to an approximate power law, $\omega^{-1 / 2}$. We observe that the deviation to this power law is larger at high pulsations. For $\phi_{0, \|}$, if the power law $\omega^{-1 / 2}$ seems to be valid at low pulsations, the deviations are very large at higher pulsations.

As an example, Fig. 6 shows the frequency dependence of $\phi_{u, \|}$ and $\phi_{u, \perp}$ at a constant velocity $U=1 \mathrm{~ms}^{-1}$. This time, we note that $\phi_{u, \|}<\phi_{u \perp}$. These two fluxes exhibit two different power laws with pulsation, $\phi_{u, \|} \sim \omega^{-1}$ and $\phi_{u, \perp}$ $\sim \omega^{-3 / 2}$. As for $\phi_{0}$, the effect of $\alpha$ can be neglected. Finally, we verify the linearity of the two components of $\mathrm{Re}_{\mathrm{m}} \phi_{u}$ with velocity at constant pulsation $\omega=3142 \mathrm{rads}^{-1}$ (inset of Fig. 6). This result has been confirmed for different pulsations in the considered range of this study. We verified that the slopes $\phi_{u, \|}(U)$ and $\phi_{u, \perp}(U)$ are independent of $\alpha$.

The study of the pulsation dependence of $\psi_{\alpha}$ for different $\alpha$ values at constant velocity has shown a non-power law behavior for the two components $\psi_{\alpha, \|}$ and $\psi_{\alpha, \perp}$ (Figs. 7 and $8)$. First, we measure that $\psi_{\alpha}(\omega)$ curves are the function of $\alpha$. The variations with the pulsation of the two components at

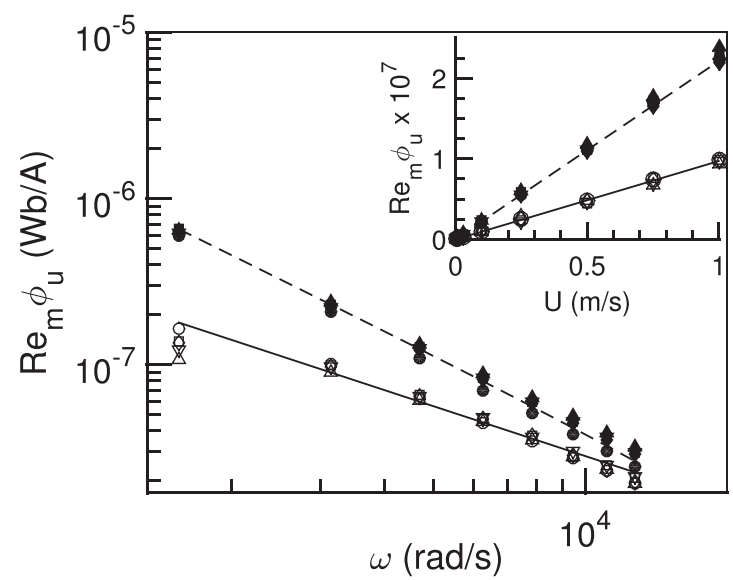

FIG. 6. $\operatorname{Re}_{\mathrm{m}} \phi_{u}$ vs $\omega$ at $U=1 \mathrm{~ms}^{-1}$ for $\alpha=0.0 \%(\bigcirc, \bigcirc), 0.3 \%(\square, \boldsymbol{\square})$, $2.0 \%(\diamond, \diamond), 4.5 \%(\nabla, \nabla), 6.9 \%(\triangle, \boldsymbol{\Delta})$, where $\operatorname{Re}_{\mathrm{m}} \phi_{u, \|}$ : unfilled symbols and $\operatorname{Re}_{\mathrm{m}} \phi_{u, \perp}$ : filled symbols. (-) represents $\omega^{-1 / 2}$ and $(--)$ represents $\omega^{-3 / 2}$. Inset $\operatorname{Re}_{\mathrm{m}} \phi_{u}$ vs $U$. 


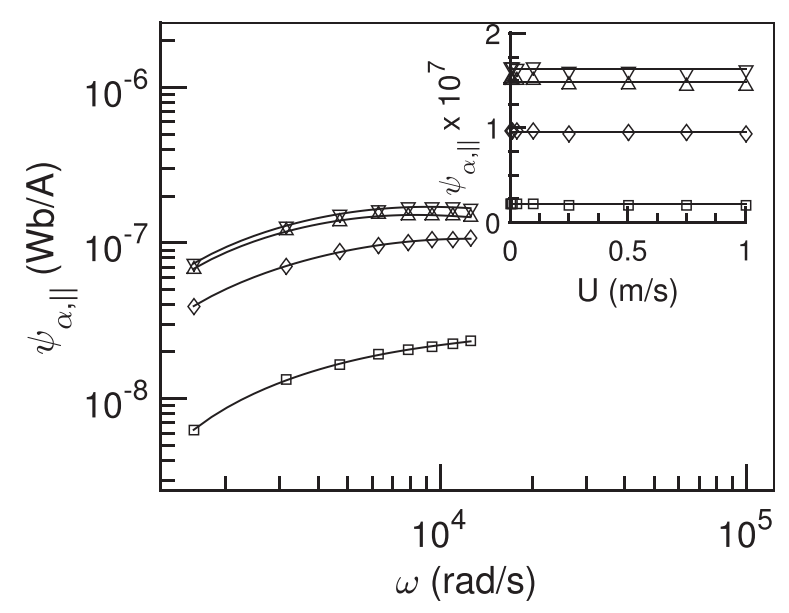

FIG. 7. $\Delta \psi_{\alpha, \|}$ vs $\omega$ at $U=1 \mathrm{~ms}^{-1}$ for $\alpha=0.3 \%(\square), 2.0 \%(\diamond), 4.5 \%(\nabla)$, and $6.9 \%(\triangle)$. Inset $\psi_{\alpha . \|}$ vs $U$.

constant $\alpha$ are really different. All the curves for $\psi_{\alpha, \perp}(\omega)$ collapse to a vertical asymptote at about $12600 \mathrm{rad} \mathrm{s}^{-1}$. Consequently, $\psi_{\alpha, \perp}$ tends to zero beyond this critical pulsation. On the other hand, $\psi_{\alpha, \|}(\omega)$ at a constant $\alpha$ increases with the pulsation and reaches a plateau value at high pulsation. Hence for $\omega>7000 \mathrm{rad} \mathrm{s}^{-1}, \psi_{\alpha, \|}$ is independent of $\omega$. The two $\psi_{\alpha}$ components are on the same order of magnitude at low pulsations and differ by more than one decade at high pulsations. This behavior is really different than those of $\phi_{0}$ and $\phi_{u}$. The velocity dependence of $\psi_{\alpha}$ are presented in the inset of Figs. 7 and 8. For both components, we observe the remarkable result that $\psi_{\alpha}$ is independent of $U$. All the curves presented before have been obtained for rod with groovetype rods. They have been confirmed with hole-type rods but these results are not presented here for the sake of brevity.

Combining the results obtained with holes and grooves geometries, the plot of $\left\|\psi_{\alpha}(\alpha)\right\|$ at a constant $U$ and $\omega$ is given in Fig. 9. We observe that the results obtained with both geometries are consistent. This is remarkable because it appears that $\left\langle\psi_{\alpha} \mid \psi_{\alpha}\right\rangle$ depends only on $\alpha$ values and not on the geometry of the voids. The experiments show that $\psi_{\alpha} \sim \alpha$ for $\alpha<4 \%$ and saturates for higher values in the range of $\alpha$ studied. This result is in agreement with literature where the authors obtained a similar curve for the fluctuations of the emf signal. ${ }^{12}$

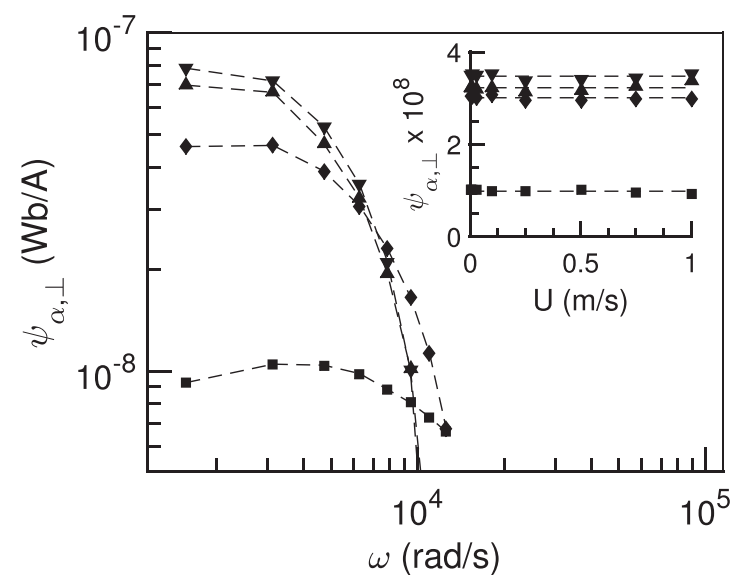

FIG. 8. $\Delta \psi_{\alpha, \perp}$ vs $\omega$ at $U=1 \mathrm{~ms}^{-1}$ for $\alpha=0.3 \%(\mathbf{\square}), 2.0 \%(\bullet), 4.5 \%(\boldsymbol{\nabla})$, and $6.9 \%(\boldsymbol{\Lambda})$. Inset $\psi_{\alpha, \perp}$ vs $U$.

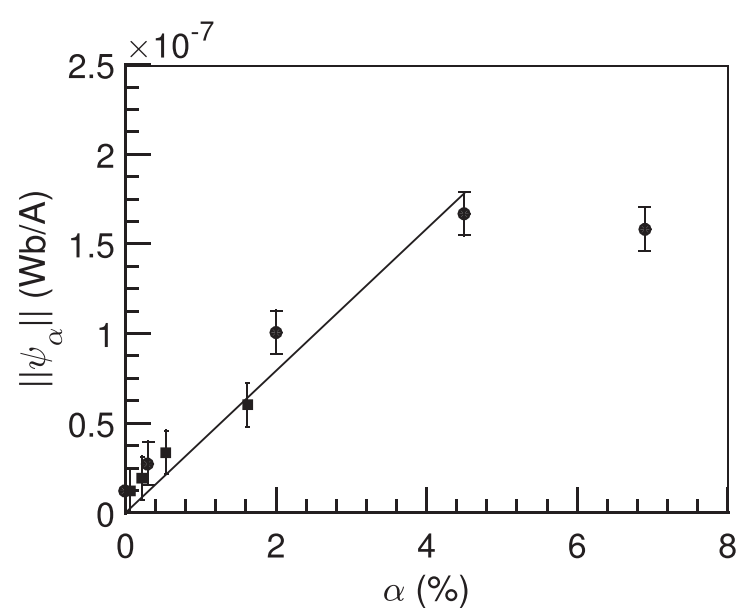

FIG. 9. $\left\|\psi_{\alpha}\right\|=\left\langle\psi_{\alpha} \mid \psi_{\alpha}\right\rangle^{1 / 2}$ vs $\alpha$ at $\omega=6280 \mathrm{rad} \mathrm{s}^{-1}$. ๑: grooves, and holes.

In order to study the coupling between the Lorentz force and the Faraday effects, we analyze the Fourier transform of $\|\Delta V\|^{2}$. For all cases, we measured $\omega_{\alpha}$ and we verified that $\omega_{\alpha}=k_{\alpha} U$ at a constant pulsation, in agreement with our model (Fig. 10). Furthermore, we check that $\omega_{\alpha}$ is independent of $\omega$ at constant $U$. In Fig. 11, $A_{1}$ is plotted with different $\alpha$ at $\omega=6283 \mathrm{rad} \mathrm{s}^{-1}$. As predicted, we observe that $A_{1}$ is a linear function of the velocity. The intercept with the vertical axis gives $\left\langle\phi_{0} \mid \psi_{\alpha}\right\rangle$ which increases linearly with $\alpha$ at low $\alpha$. The slope is $\left\langle\phi_{u} \mid \psi_{\alpha}\right\rangle$. We note that the coupling between $U$ and $\alpha$ increases with $\alpha$ and saturates as observed previously for $\psi_{\alpha}$. This last effect has been confirmed by Fourier analysis and Fig. 12 shows the independence of $\left\langle\psi_{\alpha} \mid \psi_{\alpha}\right\rangle$ with velocity. For all the experiments the values of fluxes obtained by direct processing and by Fourier transform are similar.

The analyses of all the results show clearly the validity of the model presented in Section II. In the first point, the property that the perturbed magnetic flux is independent of the void geometry confirms the validity and robustness of the model at first order. In the second point, all the experimental FFT spectra are characterized by the two first harmonic peaks, this result proves the relevance of the first order development in $\mathrm{Re}_{\mathrm{m}}$.

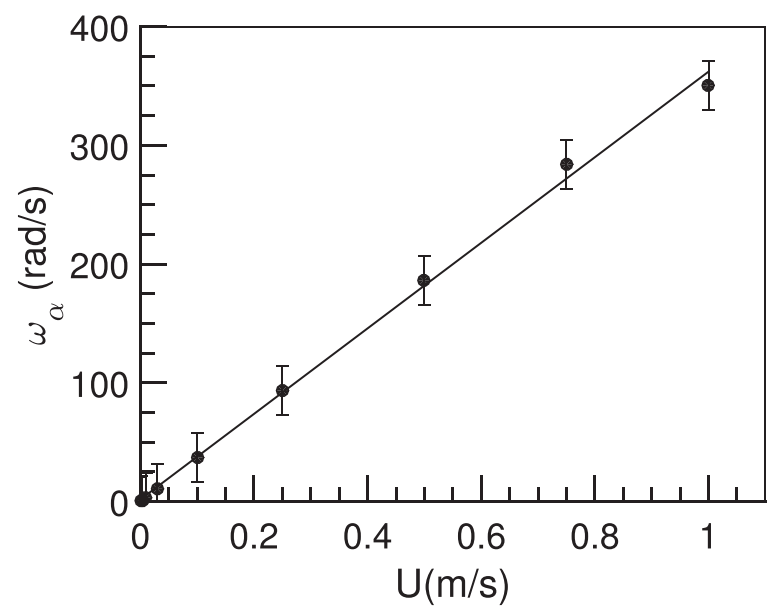

FIG. 10. $\omega_{\alpha}$ vs $U$ for $\alpha=6.9 \%$ and $\omega=6280 \mathrm{rad} \mathrm{s}^{-1}$. 


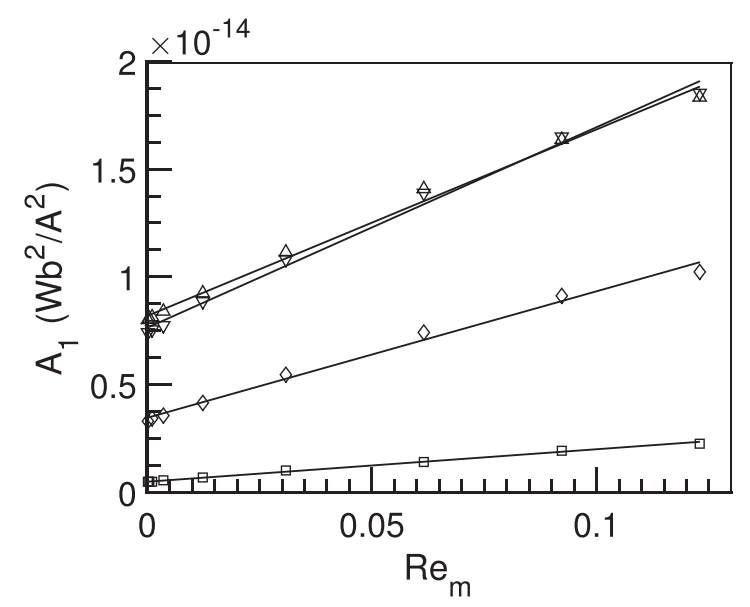

FIG. 11. $A_{1}\left(\mathrm{~Wb}^{1} \mathrm{~A}^{-2}\right)=2\left\langle\phi_{0} \mid \psi_{\alpha}\right\rangle+2 \operatorname{Re}_{\mathrm{m}}\left\langle\phi_{u} \mid \psi_{\alpha}\right\rangle$ vs $U$ for $\alpha=0.3 \%$ ( $\left.\square\right)$, $2.0 \%(\diamond), 4.5 \%(\nabla), 6.9 \%(\triangle)$, and $\omega=6280 \mathrm{rad} \mathrm{s}^{-1}$.

In the model, the choice of the characteristic length is determining. In this work where $\delta<D$, the natural characteristic length, which can be derived from the Stoke's circulation theorem for the electric field, is $\delta(\omega)$. This length can be obtained independently from the dimensional analyses of $\operatorname{Re}_{\mathrm{m}} \phi_{u}$ and $\phi_{0}$ based on the Maxwell equations. Indeed, considering that the magnetic field can be expressed as $\vec{B}=\overrightarrow{B_{0}}+\vec{B}_{u}+\vec{B}_{\alpha}$, where $B_{u}$ and $B_{\alpha}$ are the perturbation in the magnetic field due to the velocity and void fraction, respectively, we found that the ratio $B_{u} / B_{0} \sim \mathrm{Re}_{\mathrm{m}}$. We recall that $\mathrm{Re}_{\mathrm{m}}$ is defined in this study with the characteristic length scale $\delta$.

At this stage, a similar analysis based on the Maxwell equations can provide that the in-phase and quadrature-phase components are of the same order for $B_{0}$ and $B_{u}$, respectively. This analysis cannot predict the exact ratio of the two components. Numerical simulations with $\mathrm{COMSOL}^{\circledR}$ have shown that the average magnetic field in the penetration depth has an amplitude independent of the frequency. On the other hand, the effective surface for the integration of the flux is at first order $\pi D \delta$. A dimensional analysis based on

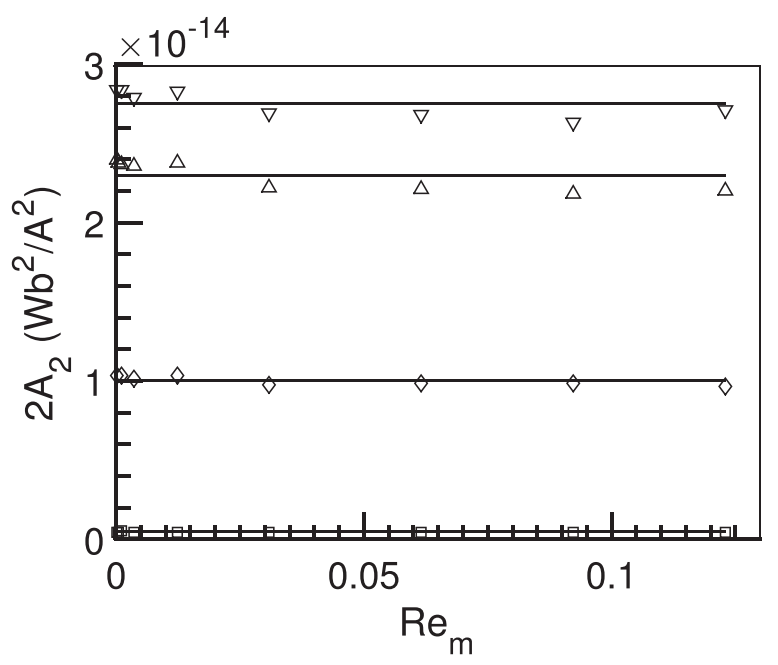

FIG. 12. $2 A_{2}\left(\mathrm{~Wb}^{1} \mathrm{~A}^{-2}\right)=\left\langle\psi_{\alpha} \mid \psi_{\alpha}\right\rangle$ vs $U$ for $\alpha=0.3 \%(\square), 2.0 \%(\diamond), 4.5 \%$ $(\nabla), 6.9 \%(\triangle)$, and $\omega=6280 \mathrm{rad} \mathrm{s}^{-1}$. the Maxwell's equations show that $\phi_{0, \perp} \sim \omega^{-1 / 2}$ and $\phi_{u, \|}$ $\sim \omega^{-1}$ are in agreement with the experiments. These scaling laws have been obtained directly by considering Faraday induction and Lorentz force effects for $\phi_{0}$ and $\phi_{u}$ separately for an exciting magnetic field in phase with the current. It is noteworthy that the scaling laws for $\phi_{0, \|}$ and $\phi_{u, \perp}$ cannot be obtained by the same approach due to the strong coupling between Maxwell-Ampère and Maxwell-Faraday equations for $\phi_{0, \|}$, and also the Lorentz force for $\phi_{u, \perp}$.

For small $\alpha$ values, $\sigma=\sigma_{0}+\sigma^{\prime} \alpha$ (following a Taylor expansion). Consequently, $\psi_{\alpha} \sim \alpha$. This linearity was observed experimentally for $\alpha \lesssim 4 \%$. For groove depth smaller than $\delta$ and for small $\alpha$ values, $\alpha$ scales as $1 / \delta(\omega)$. This relation yields to a pulsation independence of $\psi_{\alpha}$ that is observed experimentally at high pulsations.

The whole results show that the Lorentz force effects and Faraday induction effects are intimately coupled. This leads to complex properties of the total magnetic flux, which arises from the fact that the characteristic length of the magnetic flux and that of the flow are not sufficiently separated. In this work, $\delta$ is neither very small nor very large when compared to $D$. Even if the coupling effects are strong, we were able to separate $U$ and $\alpha$ effects because $\phi_{u}$ is independent of $\alpha$ and $\psi_{\alpha}$ is independent of $U$ at first order. This is valid only for small $\mathrm{Re}_{\mathrm{m}}$ and $\alpha$ values.

From a practical point of view, the Fourier analysis of $\|\Delta V\|^{2}$ is sufficient to measure simultaneously the velocity and the void fraction of a conducting liquid in large number of applications, as long as $\omega_{\alpha}$ can be defined.

\section{CONCLUSION}

In this paper, we experimentally studied the magnetic flux distortion in the two-phase liquid metal flow excited by an AC magnetic field. A specific experimental setup with a moving ECFM has been designed. The two-phase liquid metal was modeled by aluminium rods along which ECFM translates at constant velocity. The voids were simulated by machining holes and grooves on the rods. In these experiments, $\alpha$ varies between $0 \%$ and $6.9 \%$. The coil lengths are larger but comparable to $\lambda_{\alpha}$.

We have studied the effects of pulsation of exciting electrical current $\left(1.5 \times 10^{3} \leq \omega \leq 12.5 \times 10^{3} \mathrm{rad} \mathrm{s}^{-1}\right)$, of the flow velocity $\left(0 \leq U \leq 1 \mathrm{~ms}^{-1}\right)$, and of the void volume fraction on the magnetic flux distortion. In this configuration, the relative motion of aluminium simulates a plug flow. In all the experiments, the magnetic flux was demodulated with a Lockin amplifier in order to characterize its in-phase and quadrature-phase components.

The results clearly show that the distortion in the magnetic flux results from a strong coupling between Lorentz force effects and Faraday induction. They have been analyzed on the basis of a perturbative model, where the total magnetic flux is expanded at first order in $\alpha$ and $U$ : $\phi \approx \phi_{0}+\operatorname{Re}_{\mathrm{m}} \phi_{u}+\phi_{\alpha}$.

The study of the pulsation dependence of the magnetic flux points out that $\phi_{0, \|}$ and $\phi_{u, \perp}$ result from a strong coupling between Faraday induction and Lorentz force effects. On the other side, $\phi_{0, \perp}$ and $\phi_{u, \|}$ seem to depend mainly on 
the Faraday induction for the first and on Lorentz force for the second. Indeed, the scaling laws $\phi_{0, \perp} \sim \omega^{-\frac{1}{2}}$ and $\phi_{u, \|} \sim \omega^{-1}$ can be deduced directly from the Maxwell-Faraday and Maxwell-Ampere equations, respectively. An important result is that $\operatorname{Re}_{\mathrm{m}} \phi_{u}$ is linear in $U$ and independent of $\alpha . \psi_{\alpha}$ is independent of $U$ and linear in $\alpha$. This experimental result can be justified theoretically because $\operatorname{Re}_{\mathrm{m}}$ and $\alpha$ are small. It has been obtained for all the void geometries.

For practical applications, the Fourier analysis of the demodulated voltage difference between the two secondary coils $\|\Delta V\|^{2}$ allows to measure the velocity and the void fraction. Indeed, the first peak of $\|\Delta V\|^{2}$ FFT increases linearly with $U$. Hence, the second peak is proportional to $\alpha^{2}$. This approach can be used after calibration.

At this stage, the coupling between Faraday induction and the Lorentz force effects is not well understood. A theoretical model should be developed to calculate the Eddy current distortions due to the flow and the void at the local scale and their contributions on the magnetic flux at the flow scale.

\section{ACKNOWLEDGMENTS}

The authors acknowledge Hervé Ayroles and Grégory Ehses at IMFT Toulouse for their technical assistance and Emeryk Ablonet for contributions in experiments. This work has been financially supported by CEA, Cadarache.
${ }^{1}$ J. C. Maxwell, A Treatise on Electricity And Magnetism: Unabridged 3rd (Dover Publications, New York, 1954).

${ }^{2}$ N. Tanatugu, Y. Fujii-e, and T. Suita, J. Nucl. Sci. Technol. 9(12), 753 (1972).

${ }^{3}$ K. Nakamoto, S. Tamura, K. Ishii, H. Kuwahara, N. Ohyama, and T. Muramatsu, Nucl. Eng. Des. 82, 393 (1984).

${ }^{4}$ N. Terzija, W. Yin, G. Gerbeth, F. Stefani, K. Timmel, T. Wondrak, and A. Peyton, Meas. Sci. Technol. 22(1), 015501 (2011).

${ }^{5}$ R. C. Baker, Prog. Nucl. Energy 1, 41 (1977).

${ }^{6}$ J. R. Bowler, J. Appl. Phys. 75(12), 8128 (1994).

${ }^{7}$ J. R. Bowler, S. J. Norton, and D. J. Harrison, J. Appl. Phys. 75(12), 8138 (1994).

${ }^{8}$ J. A. Shercliff, The Theory of Electromagnetic Flow-Measurement (University Press, Cambridge, 1962).

${ }^{9}$ J. Hemp, J. Phys. D: Appl. Phys. 24(3), 244 (1991).

${ }^{10}$ J.-E. Cha, Y.-C. Ahn, K.-W. Seo, H. Y. Nam, J. H. Choi, and M. H. Kim, J. Nucl. Sci. Technol. 40(10) 744 (2003).

${ }^{11}$ D. G. Wyatt, Int. J. Multiphase Flow 12(6), 1009 (1986).

${ }^{12}$ T. Ogino, H. Inujima, and K. Haga, J. Nucl. Sci. Technol. 21(3), 172 (1984).

${ }^{13}$ J. Fröhlich, S. Schwarz, S. Heitkam, C. Santarelli, C. Zhang, T. Vogt, S. Boden, A. Andruszkiewicz, K. Eckert, S. Odenbach et al., Eur. Phys. J. Spec. Top. 220(1), 167 (2013).

${ }^{14}$ C. Zhang, S. Eckert, and G. Gerbeth, Int. J. Multiphase Flow 31(7), 824 (2005).

${ }^{15}$ S. Shin and I. Kang, Int. J. Multiphase Flow 28(1), 105 (2002)

${ }^{16}$ A. Serizawa, T. Ida, O. Takahashi, and I. Michiyoshi, Int. J. Multiphase Flow 16(5), 761 (1990).

${ }^{17}$ S. Eckert, G. Gerbeth, and O. Lielausis, Int. J. Multiphase Flow 26(1), 45 (2000).

${ }^{18}$ S. Eckert, G. Gerbeth, and O. Lielausis, Int. J. Multiphase Flow 26(1), 67 (2000).

${ }^{19}$ S. Schwarz and J. Fröhlich, Int. J. Multiphase Flow 62, 134 (2014).

${ }^{20}$ J. Zhang and M. J. Ni, Phys. Fluids 26(10), 102102 (2014).

${ }^{21}$ M. Kumar, Ph. Tordjeman, W. Bergez, and M. Cavaro, Rev. Sci. Instrum. 86(10), 106104 (2015). 